\title{
FACTORES DE RIESGO RELACIONADOS AL BULLYING EN LOS ESTUDIANTES DE LA UNIVERSIDAD NACIONAL JOSÉ FAUSTINO SÁNCHEZ CARRIÓN
}

\author{
RISK FACTORS RELATED TO BULLYING \\ IN STUDENTS OF JOSÉ FAUSTINO SÁNCHEZ CARRIÓN UNIVERSITY
}

\author{
Recibido: 13/04/2015 Revisado: 08/05/2015 Aceptado: 02/06/2015
}

\section{Mirtha Sussan Trejo López', Yolanda Marianela Castañeda Carrión', Cosme Ulises Valverde Flores', Miguel Angel Aguilar Luna Victoria ${ }^{1}$}

\begin{abstract}
RESUMEN
Objetivo: Identificar los factores de riesgo significativos del bullying en los estudiantes de la Universidad Nacional José Faustino Sánchez Carrión de Huacho. Métodos: La población estuvo conformada por 11000 estudiantes matriculados en el semestre académico 2014-I en la Sede de Huacho de las 12 Facultades y se tomó un muestreo aleatorio simple de 260 estudiantes. Los instrumentos utilizados para la recopilación de datos fueron, el bullying la escala de Lyn Quine adecuado al contexto universitario mediante una escala de autoreporte, según Quine (2003) esta escala permite estimar el comportamiento persistente, ofensivo, abusivo intimidatorio, malicioso o insultante, abuso de poder lo cual hace que el receptor se sienta perturbado, amenazado, humillado o vulnerable afectando su integridad personal y para la disfunción familiar se empleó la ficha técnica del Doctor Smilkstein, Gabriel evaluando cinco funciones básicas: adaptación, participación, ganancia, afecto y recursos. Resultados: El $43,1 \%$ fueron estudiantes varones y $56,9 \%$ mujeres; sus edades estuvieron comprendidas entre 16 y 27 años siendo el promedio 19 años, el 21,2 \% de los estudiantes han sufrido de bullying y el nivel de disfunción familiar distribuido: Grave, 10,8\%; Leve con un $29,2 \%$ y Familia Funcional con 60,0\%. Conclusiones: La edad está relacionada con la presencia del bullying verbal y la disfunción familiar con la presencia del bullying psicológico con un nivel de confianza del $95 \%$. El factor de riesgo asociado al bullying en el género femenino fue la disfunción familiar.
\end{abstract}

Palabras clave: Bullying, disfunción familiar, factores de riesgo

\section{ABSTRACT}

Objective: To identify significant risk factors of bullying on students of the National José Faustino Sanchez Carrion University of Huacho. Methods: The population consisted of 11,000 students enrolled in the semester 2014-I at Huacho'ssede of the 12 faculties and a random sample of 260 students was taken. The instruments used for data collection were, bullying Quine of the Lyn scale, appropriate to the university context using a self-report scale, according Quine (2003), this scale to estimate the persistent, offensive, abusive, intimidating behavior, malicious or insulting abuse power which makes the student feel upset, threatened, humiliated or vulnerable affected their personal integrity and in order to family dysfunction, sheet of Doctor Smilkstein Gabriel was used, evaluating five basic functions: adaptation, participation, gain, affection and resources. Results: $43,1 \%$ were male and 56,9\% female students; their ages were between 16 and 27 years with an average 19 years, $21,2 \%$ of students have suffered from bullying and level of family dysfunction distributed: Grave, 10,8\%; Mild with $29,2 \%$ and $60,0 \%$ Functional Family. Conclusions: The age is related to the presence of verbal bullying and family dysfunction in the presence of psychological bullying with a confidence level of $95 \%$ is applied. The risk factor associated with bullying in females was family dysfunction.

Keywords: Bullying, familiar disfunction, risk factor.

\section{INTRODUCCIÓN}

El bullying es una modalidad de agresión no encubierta y hace referencia a comportamientos hostiles de un alumno o un grupo de alumnos hacia la víctima, ocasionándoles daños irreparables en su autoestima y otras áreas de su desarrollo personal. Sweeting y West (2001, véase Stan, 2009) encontraron que "...los jóvenes que son menos atractivos físicamente, que tienen sobrepeso, alguna discapacidad (problemas de visión, oído o habla) o un bajo rendimiento académico, son más propensos a ser intimidados". Desde esta perspectiva se puede afirmar que muchos de los agresores resaltan constantemente los defectos físicos de sus compañeros con la finalidad de ridiculizarle y humillarle ante los demás.

En la actualidad vivimos una época marcada por la incertidumbre, la crisis de valores, las carencias afectivas, las familias quebradas, el egoísmo y el vacío existencial, unido paralelamente a una sobrevaloración de la tecnología, hasta el punto que sea ésta la que en muchos sentidos gobierne nuestras vidas. El colegio o la institución atraviesa una de sus etapas más problemáticas debido probablemente a que ha dejado de ser el lugar seguro y confiable donde los niños, adolescentes y jóvenes van a aprender y a desarrollarse cognitiva, social y afectivamente, para convertirse en un lugar donde se aprende lo que es el maltrato entre iguales, la humillación, la discriminación, la exclusión social, entre otras situaciones que a la larga configuran en la persona no sólo que es víctima, sino también espectador o agresor, un futuro incierto, donde probablemente ciertas conductas desadaptativas pueden verse instaladas, desde la simple pero altamente cuestionable indiferencia social, hasta conductas altamente violentas.

Varios estudios muestran que, a menudo, los profesores se crean expectativas, positivas o negativas, respecto a sus alumnos e interactúan en público más frecuentemente con los estudiantes de expectativa positiva. Esto da como resultado que haya un grupo pequeño de alumnos más "brillantes" que intervienen casi siempre y otro pequeño grupo de alumnos más "lentos" que no participan casi nunca. Además, los estudiantes de altas expectativas suelen 
recibir muchos elogios, y, los de bajas expectativas, muchas críticas. Así, la motivación de estos últimos disminuye y se siente discriminados respecto al resto de la clase.

Muchos profesores no saben cómo afrontar cuando atacan a un alumno dentro de su aula de clases. La palabra bullying describe un modo de trato entre personas. Su significado fundamental es: acosar, molestar, hostigar, obstaculizar. Con frecuencia los periódicos nos conmueven o asustan con noticias trágicas o alarmantes, pero conociendo el poder amplificador de los medios, tenemos que preguntarnos: ¿Cuál es la gravedad real del problema? ¿Sabemos qué hacer para atacarlo? ¿Lo estamos haciendo?

Es un problema que se está haciendo dramáticamente actual y muy preocupante en nuestra sociedad, muchas veces no es desinterés lo que le inhibe, es que no saben qué hacer, o no se atreven a hacerlo. Las excusas que se dan son siempre las mismas: es sólo una broma inofensiva, los niños o jóvenes deben aprender a soportar esos conflictos, todo ello forma parte del crecimiento, tienen que aprender a librar sus propias batallas, todos hemos pasado por situaciones parecidas.

En las investigaciones realizadas en nuestro país por la Comisión Nacional para el Desarrollo y Vida sin Drogas (DEVIDA), en el año 2007 a nivel nacional, se reportaron que el $40 \%$ del total de escolares secundarios del Perú son víctimas de agresiones en las modalidades de ignorados (28\%), excluidos $(22 \%)$, discriminados $(21 \%)$, físicamente agredidos (24\%) y sexualmente acosados $(10 \%)$.

Del mismo modo, Becerra, Flores y Vásquez (2009) hallaron que el $45 \%$ de los alumnos de los colegios de Lima Metropolitana eran víctimas de agresión y la modalidad más frecuente era la agresión verbal $(67 \%)$.

Por la ONG el Plan Internacional América Latina es la región del mundo con mayor número de casos de bullying. Las causas de práctica se vinculan con la violencia y la desigualdad que viven los más abusadores. En América Latina el $70 \%$ de los niños son directamente o indirectamente afectados por el bullying.

La importancia de realizar esta investigación es indagar la situación universitaria de los estudiantes y tomar medidas preventivas o correctivas sobre aquellos factores de riesgos relacionados a la presencia del bullying que pueden dar paso a la violencia dentro de los estudiantes para brindarle bienestar a los estudiantes y salvaguardar la integridad de los mismos que son del sector más susceptible de caer o crear actos de violencia, aportando conocimientos para la mejora de convivencia y la promoción de actitudes positivas de los jóvenes.

En cuanto a los docentes es crear conciencia sobre el papel formativo y la trascendencia que recobren los jóvenes estudiantes universitarios, así como también prever dentro de esa noble tarea aquellas medidas que frenen la violencia y puedan crear una universidad segura, un espacio de formación integral donde se establezcan relaciones emocionales sanas y plenas.

La hipótesis planteada fue que los factores de riesgo están relacionados significativamente con el bullying en los estudiantes de la Universidad Nacional José Faustino Sánchez Carrión y el objetivo identificar si la edad, género y disfunción familiar están relacionados en la presencia del bullying verbal, físico y psicológico.

\section{MATERIAL Y MÉTODOS}

Esta investigación tiene características de estudio transversal y comparativo, los encuestados fueron estudiantes de la Universidad Nacional José Faustino Sánchez Carrión, elegidos mediante muestreo aleatorio simple, iniciándose previamente con el desarrollo de un marco teórico y utilizando como instrumentos dos encuestas, con cuestionarios aplicados en el mismo instante a los participantes, uno para conocer efectivamente el bullying en el contexto universitario mediante la escala de Lyn Quine, y otro orientado a conocer el grado de disfunción familiar mediante la ficha técnica del Doctor Gabriel Smilkstein, ambos cuestionarios ampliamente utilizados a nivel internacional.

Las respuestas resultantes de esta aplicación fueron codificadas y analizadas con el fin de determinar claridad, coherencia y pertinencia de las mismas a fin de obtener sinceridad en sus declaraciones. Cada uno de los ítems consta de un enunciado y cinco opciones de respuesta, entre estas posibilidades el estudiante debió seleccionar una única respuesta. Finalmente se descartaron 6 cuestionarios por error o falsedad de algunas de sus respuestas.

Las variables estudiadas fueron: principal presencia del bullying, definida como riesgo de conductas de los estudiantes, las cuales pueden ser física, verbal o psicológica; el puntaje total de ellas brinda el puntaje para el bullying general. Las variables edad, sexo, disfunción familiar, fueron las predictoras. Se utilizó la estadística descriptiva para las variables demográficas, y la prueba chi-cuadrado para comparar la edad, el sexo y la disfunción familiar con la presencia de bullying en sus diferentes formas físico, verbal y psicológico; asimismo se utilizó la regresión logística, para conocer si en conjunto las variables edad, género y disfunción familiar previamente categorizadas influyen en la presencia del bullying. Posteriormente se realizó el mismo estudio logístico pero separadamente por sexo. Las variables predictoras o independientes, se clasificaron de acuerdo a su puntaje siguiendo las recomendaciones de los especialistas y autores de los respectivos instrumentos de la siguiente manera:

Edad: Adolescencia media (16 a 19 años), Adulto joven (20 a 24 años) y Adulto medio ( 25 a 30 años). Disfunción Familiar: Grave ( 5 a 8 puntos), Leve ( 9 a 11 puntos) y Familia funcional (12 a 15 puntos). Bullying Verbal: Alto (12 a 15 puntos), Moderado (8 a 11 puntos) y Bajo (5 a 7 puntos). Bullying Físico: Alto (7 a 9 puntos), Moderado ( 5 a 6 puntos) y Bajo ( 3 a 4 puntos). Bullying Psicológico: Alto (24 a 30 puntos), Moderado (17 a 23 puntos) y Bajo (10 a 16 puntos). Bullying General: Alto (42 a 54 puntos), Moderado (30 a 41 puntos) y Bajo (18 a 29 puntos)

El análisis estadístico se realizó con el programa SPSS versión 20.

\section{Muestra}

La población estuvo conformada por 11000 estudiantes de las 12 Facultades y se tomó un muestreo aleatorio simple con un nivel de confianza del $95 \%$, esta muestra estuvo constituida por 266 estudiantes que corresponden a una proporción del $23 \%$ de la población, de los cuales 6 se descartaron por error en el llenado de su cuestionario, quedando 260 . El marco de muestreo estuvo constituido por la relación de estudiantes matriculados de cada Facultad, proporcionado por la Oficina de Registros y Asuntos Académicos de la Universidad. Se aseguró el anonimato de los estudiantes, solicitándole a cada uno de ellos su respectivo consentimiento, para cumplir con la declaración de Helsinky sobre ética de investigación.

\section{RESULTADOS}

Un total de 260 estudiantes participaron del estudio contestando los cuestionarios, de los cuales el $43,1 \%$ fueron estudiantes varones y $56,9 \%$ mujeres; sus edades estuvieron comprendidas entre 16 y 27 años siendo el promedio 19 años. El estudio indica que el $78,8 \%$ no ha sufrido bullying durante su vida universitaria, el $21,2 \%$ si ha sufrido alguna forma de bullying: el $10,0 \%$ han sido hombres y el $11,2 \%$ mujeres.

Asimismo, en cuanto al grupo de edad, los que han sufrido bullying son principalmente los que clasifican en adolescencia media con un $15,8 \%$, le siguen adulto joven con un $4,2 \%$ y adulto medio con un $1,2 \%$.

El grupo con mayor porcentaje fue el de testigo con un $75,0 \%$ distribuido en un $30,4 \%$ y $44,6 \%$ entre hombres y mujeres respectivamente. Entre testigo y víctima siempre ha sido el grupo con mayor psicopatología femenino tal como se muestra en la tabla 1. 
Tabla 1. Distribución de los alumnos clasificados según su rol en el bullying y género

\begin{tabular}{|c|c|c|c|c|c|c|}
\hline \multirow{3}{*}{$\begin{array}{l}\text { ROL EN EL } \\
\text { BULLYING }\end{array}$} & \multicolumn{4}{|c|}{ Género } & \multicolumn{2}{|c|}{ TOTAL } \\
\hline & \multicolumn{2}{|c|}{ Masculino } & \multicolumn{2}{|c|}{ Femenino } & \multirow[t]{2}{*}{$\mathbf{N}^{\circ}$} & \multirow[t]{2}{*}{$\%$} \\
\hline & $\mathbf{N}^{\circ}$ & $\%$ & $\mathbf{N}^{\circ}$ & $\%$ & & \\
\hline Testigo & 79 & 30,4 & 116 & 44,6 & 195 & 75,0 \\
\hline Víctima & 26 & 10,0 & 29 & 11,2 & 55 & 21,2 \\
\hline Agresor & 7 & 2,7 & 3 & 1,1 & 10 & 3,8 \\
\hline TOTAL & 112 & 43,1 & 148 & 56,9 & 260 & 100,0 \\
\hline
\end{tabular}

Fuente: Elaboración propia

Tabla 2. Distribución de los alumnos clasificados según disfunción familiar y bullying psicológico

\begin{tabular}{|c|c|c|c|c|c|c|c|c|}
\hline \multirow{3}{*}{$\begin{array}{l}\text { DISFUNCIÓN } \\
\text { FAMILIAR }\end{array}$} & \multicolumn{6}{|c|}{ BULLYING PSICOLÓGICO } & \multicolumn{2}{|c|}{ TOTAL } \\
\hline & \multicolumn{2}{|c|}{ Bajo } & \multicolumn{2}{|c|}{ Moderado } & \multicolumn{2}{|c|}{ Alto } & \multirow[b]{2}{*}{$\mathbf{N}^{\circ}$} & \multirow[b]{2}{*}{$\%$} \\
\hline & $\mathbf{N}^{\circ}$ & $\%$ & $\mathbf{N}^{\circ}$ & $\%$ & $\mathbf{N}^{\circ}$ & $\%$ & & \\
\hline Grave & 0 & 0,0 & 7 & 2,7 & 21 & 8,1 & 28 & 10,8 \\
\hline Leve & 71 & 27,3 & 5 & 1,9 & 0 & 0,0 & 76 & 29,2 \\
\hline Familia funcional & 132 & 50,8 & 22 & 8,5 & 2 & 0,8 & 156 & 60,0 \\
\hline TOTAL & 203 & 78,1 & 34 & 13,1 & 23 & 8,8 & 260 & 100,0 \\
\hline
\end{tabular}

Fuente: Elaboración propia

Tabla 3. Distribución de los alumnos clasificados según grupo de edad y bullying verbal

\begin{tabular}{|c|c|c|c|c|c|c|c|c|}
\hline \multirow[t]{3}{*}{ GRUPO EDAD } & \multicolumn{6}{|c|}{ BULLYING VERBAL } & \multicolumn{2}{|c|}{ TOTAL } \\
\hline & \multicolumn{2}{|c|}{ Bajo } & \multicolumn{2}{|c|}{ Moderado } & \multicolumn{2}{|c|}{ Alto } & & \\
\hline & $\mathbf{N}^{\circ}$ & $\%$ & $\mathbf{N}^{\circ}$ & $\%$ & $\mathbf{N}^{\circ}$ & $\%$ & $\mathbf{N}^{\circ}$ & $\%$ \\
\hline Adolescencia media & 36 & 13,8 & 119 & 45,8 & 24 & 9,2 & 179 & 68,8 \\
\hline Adulto joven & 24 & 9,2 & 33 & 12,7 & 4 & 1,5 & 61 & 23,5 \\
\hline Adulto medio & 10 & 3,8 & 9 & 3,5 & 1 & 0,4 & 20 & 7,7 \\
\hline TOTAL & 70 & 26,9 & 161 & 61,9 & 29 & 11,2 & 260 & 100,0 \\
\hline
\end{tabular}

Fuente: Elaboración propia

Tabla 4. Distribución de los alumnos clasificados según género y bullying físico

\begin{tabular}{|c|c|c|c|c|c|c|c|c|}
\hline \multirow[t]{3}{*}{ GÉNERO } & \multicolumn{6}{|c|}{ BULLYING FísICO } & \multirow{2}{*}{\multicolumn{2}{|c|}{ TOTAL }} \\
\hline & \multicolumn{2}{|c|}{ Bajo } & \multicolumn{2}{|c|}{ Moderado } & \multicolumn{2}{|c|}{ Alto } & & \\
\hline & $\mathbf{N}^{\circ}$ & $\%$ & $\mathbf{N}^{\circ}$ & $\%$ & $\mathbf{N}^{\circ}$ & $\%$ & $\mathbf{N}^{\circ}$ & $\%$ \\
\hline $\begin{array}{l}\text { Masculino } \\
\text { Femenino } \\
\text { TOTAL }\end{array}$ & $\begin{array}{r}88 \\
118 \\
206\end{array}$ & $\begin{array}{l}33,8 \\
45,4 \\
79,2\end{array}$ & $\begin{array}{l}20 \\
25 \\
45\end{array}$ & $\begin{array}{r}7,7 \\
9,6 \\
17,3\end{array}$ & $\begin{array}{l}4 \\
5 \\
9\end{array}$ & $\begin{array}{l}1,6 \\
1,9 \\
3,5\end{array}$ & $\begin{array}{l}112 \\
148 \\
260\end{array}$ & $\begin{array}{r}43,1 \\
56,9 \\
100,0\end{array}$ \\
\hline
\end{tabular}

Fuente: Elaboración propia

Tabla 5. Análisis de las variables predictoras usando la chi-cuadrado

\begin{tabular}{lrc}
\hline VARIABLE & \multicolumn{2}{c}{ CHI-CUADRADO } \\
\cline { 2 - 3 } PREDICTORA & \multicolumn{1}{c}{ Valor } & Significancia \\
\hline Edad & 15,315 & 0,004 \\
Género & 0,052 & 0,0974 \\
Disfunción familiar & 185,964 & 0,000 \\
\hline
\end{tabular}

Fuente: Elaboración propia
Tabla 6. Análisis de las variables predictoras usando la regresión logística para el género masculino

\begin{tabular}{lll}
\hline VARIABLE & g.l. & Sig. \\
PREDICTORA & & \\
\hline Disfunción familiar & 1 & 0,976 \\
Edad & 1 & 0,629 \\
Constante & 1 & 0,000 \\
\hline
\end{tabular}

Fuente: Elaboración propia 
Tabla 7. Análisis de la variable predictora usando la regresión logística, para el género femenino

\begin{tabular}{lllllcr}
\hline $\begin{array}{l}\text { VARIABLE } \\
\text { PREDICTORA }\end{array}$ & $\beta$ & g.I. & Sig. & $e^{\beta}$ & \multicolumn{2}{c}{$\begin{array}{c}\text { INTERVALOS DE CONFIANZA } \\
\text { PARA }\end{array}$} \\
\cline { 3 - 6 } & & & & & INFERIOR & SUPERIOR \\
\hline Disfunción familiar(1) & $-1,069$ & 1 & 0,048 & 0,343 & 0,119 & 0,991 \\
Constante & $-0,357$ & 1 & 0,469 & 0,700 & & \\
\hline
\end{tabular}

Fuente: Elaboración propia

\section{DISCUSIÓN}

El funcionamiento familiar está en relación directa con la presencia del bullying psicológico, hay un $8,1 \%$ de alumnos pertenecientes a familias disfuncionales graves y con un nivel alto de presencia de bullying psicológico, mientras que un alumno perteneciente a una familia funcional, apenas está en un $0,8 \%$.

De igual manera el bullying verbal alto aparece mayormente en el grupo de edad de adolescencia media con un $9,2 \%$, mientras que en el adulto medio sólo $0,4 \%$. En cuanto al bullying físico sólo se tiene un 3,5\%, distribuyéndose en 1,6\% para hombres y $1,9 \%$ para mujeres.

Al realizar la prueba chi-cuadrado, se observó una asociación estadísticamente significativa a un nivel del 10\%, entre las variables predictoras: grupo de edad, género y disfunción familiar, cada una por separado, diferentes a lo reportado por Pérez (2005), siendo la disfunción familiar la variable más significativa que son similares a los hallados por Hernández y Gutiérrez (2013), como se muestra en la tabla 5.

Una explicación plausible del riesgo de ser víctima de bullying a partir de la disfunción familiar podría estar dado por el hecho que el estar expuesto a fenómenos violentos en el seno familiar haría que el niño aprenda a desarrollar una conducta sumisa ante el abuso de poder de los otros.

Al igual que el estudio (Hoyos, Romero, Alega y Molinares, 2009) y (Chapell et al.,2004), es mayor el porcentaje de los alumnos que se declaran como testigos, seguido en muchos casos de las víctimas y agresores, éstos dos últimos tanto en universitarios como en escolares, con menor incidencia que los testigos.

El $21,2 \%$ de los alumnos han sufrido bullying lo que concuerda cifras similares a lo reportado por Cassiani-Miranda (2014) y Méndez (2012).

A través del análisis de regresión logística, se observa que no existe asociación significativa entre el conjunto de variables predictoras disfunción familiar y edad respecto a la presencia de bullying general, como se observa en la tabla 6 . Es necesario indicar que al hacer una diferenciación del estudio por género, el resultado indica para el caso de los estudiantes varones igualmente la disfunción familiar y grupo de edad no influyen en la presencia del bullying; sin embargo, para el caso del género femenino, la disfunción familiar es influyente en la presencia del bullying a un nivel de significancia del $5 \%$ $(p=0,048)$.

Siguiendo con el análisis para el género femenino, $e^{\beta}=0,343$ nos indica que la disfunción familiar leve (1) y funcionamiento familiar (2) son categorías protectoras respecto a la disfunción familiar grave para la aparición del bullying; es decir, las familias funcionales tienen un $65,7 \%$ más de protección respecto a las familias disfuncionales frente al bullying lo que está de acuerdo con lo afirmado por Hernández y Gutiérrez (2013).

\section{REFERENCIAS BIBLIOGRÁFICAS}

Becerra, F.; Flores, V. y Vásquez, J. (2009). Acoso escolar (bullying) en Lima Metropolitana. Texto no publicado. Universidad Federico Villarreal

Cassiani-Miranda, C., Gómez-Alhach, J., Cubides-Munévar, A. y Hernández-Carrillo, M. (2014).Prevalencia de bullying y factores relacionados en estudiantes de bachillerato de una institución educativa de Cali, Colombia, 2014. Revista de Salud Pública. 16(1).

Comisión Nacional para el Desarrollo y Vida sin Drogas: DEVIDA (2007). Estudio Nacional: Prevención y consumo de drogas en estudiantes secundarios 2007. $\mathrm{R}$ e c u p e r a d o d e : htppp://www.devida.gob.pe/Documentacion/documento sdisponibles/II_Estudio_Regional_EscolaresSec_2007. pdf

Chapell, M.,Casey, D., De La Cruz, C., Ferrell, J., Forman, J., Lipkin, R. et.al,(2004). Bullying inColegebystudents and teachers. Adolescence, 39, 53-64.

Estudio del Plan Internacional de América Latina. Acoso escolar en América Latina. Bullying en América Latina. Disponible en: www.plan americas org www.altoalbullying.com www.aprendesinmiedo.com www.bibliotecaplan.org

Hernández, M. y Gutiérrez, M. (2013). Factores de riesgo asociados a la intimidación escolar en instituciones educativas públicas de cuatro municipios del Valle del Cauca Año 2009. Revista Colombiana de Psiquiatría, 42(3), 238-247.

Hoyos, O., Olmos, K., Valega, S. y Molinares, C. (2009). El maltrato entre iguales por abuso de poder y exclusión social en estudiantes de una universidad privada de la ciudad de Barranquilla. Revista Pensamiento Psicológico, 6(13),109-126.

Méndez, M. (2012). Variables de conducta, factores de riesgo para la Salud y adaptación integral relacionados con la problemática bullying en estudiantes de educación secundaria 2012. Tesis para optar el Grado de Doctor en Psicología. España: Universidad de Murcia.

Pérez, C. (2005). Relación entre el Sexo, la Edad, el Nivel Socioeconómico y el Maltrato (Bullying) y su efecto en la presencia de Síntomas de Ansiedad y Depresión en Estudiantes Universitarios. Tesis para optar el Título de Especialista en Psicología Clínica Comunitaria. Venezuela: Universidad Católica Andrés Bello.

Quine, L. (2003). Escala de Bullying.

Stand, D. (2009). Crecer sin miedo: Estrategias positivas para controlar el acoso escolar o bullying. Colombia.

Torres, M. (2010). El problema de la violencia entre universitarios abordado desde el enfoque de la Investigación-Participación-Acción. Investigación Universitaria Multidisciplinaria, 9(9),27-36. 